\title{
Optimal sizing and placement of distribution grid connected battery systems through an SOCP optimal power flow algorithm
}

\author{
Etta Grover-Silva ${ }^{1,2}$, Robin Girard ${ }^{1}$, George Kariniotakis ${ }^{1}$
}

\begin{abstract}
The high variability and uncertainty introduced into modern electrical distribution systems due to decentralized renewable energy generators requires new solutions for grid management and power quality assurance. One of these possible solutions includes grid integrated energy storage. The appropriate size and placement of decentralized storage is highly dependent on purpose of the battery system and expected operational strategy. However, battery operational strategies are difficult to simulate simultaneously during a sizing and placement planning calculation. The motivation of this paper is to propose an algorithm that is capable of integrating sizing, placement and operational strategies of batteries into an Optimal Power Flow (OPF) distribution grid planning tool. The choice of the OPF approach permits to account for grid constraints which is more adapted for grid-connected storage devices compared to other approaches in the state of the art that are based only on an email balance analysis. This paper presents an alternating current (AC) multi-temporal OPF algorithm that uses a convex relaxation of the power flow equations to guarantee exact and optimal solutions with high algorithmic performance. The algorithm is unique and innovative due to the fact that it combines the simultaneous optimization of placement and sizing of storage devices taking into account load curves, photovoltaic (PV) production profiles, and distribution grid power quality constraints. The choice to invest in battery capacity is highly sensitive to the price of battery systems. The investment in battery systems solely for reducing losses an operational costs was proven not to be cost effective, however when battery systems are allowed to buy and sell electricity based on variable market prices they become cost effective. The assumptions used for this study shows that current battery system prices are too high to be cost effective even when allowing battery system market participation.
\end{abstract}

Keywords: Optimal power flow, storage, smart grids, renewable energy, distribution grid planning

\section{Nomenclature}

\section{Parameters}

$\eta_{s t}$

Charging and discharging efficiency of the battery system

$\bar{P}_{p v, j, t}$ Ideal PV production for node $j$ at time step $t$

$\bar{S}_{p v, j, t}$ PV maximum apparent power flow at node $j$

$\bar{V}_{j} \quad$ Voltage maximum at node $n$

$\underline{V}_{j} \quad$ Voltage minimum at node $n$

$c_{s t}^{i n v} \quad$ Investment costs of the battery system for the nominal capacity in $€ / M W h$-day

Email addresses: etta.grover-silva@mines-paristech.fr (Etta Grover-Silva), robin.girard@mines-paristech.fr (Robin Girard), georges.kariniotakis@mines-paristech.fr (George Kariniotakis)

${ }^{1}$ MINES ParisTech, PSL Research University, PERSEE - Center for Processes, Renewable Energies and Energy Systems, France

${ }^{2}$ ADEME - French Environment and Energy Management Agency, France $c_{s t}^{o m} \quad$ Operations and maintenance costs of using the battery system for the power use in $€ / M W h$-day

$c_{e, t} \quad$ Price of electricity at time step $t$

$I^{\max } \quad$ Total capital cost limit of project

$P_{l d, j, t} \quad$ Active power load at node $j$

$Q_{l d, j, t}$ Reactive power load at node $j$

$r_{j k} \quad$ Resistance of branch $j k$

$t \quad$ Duration of timestep

$x_{j k} \quad$ Reactance of branch $j k$

Sets

$J \quad$ Set of all nodes $j \in J$

$J_{s t} \quad$ Set of nodes chosen for battery placement $j \in J_{s t}$

\section{Variables}

$\ell_{j k, t} \quad$ Square of current in branch $j k$

$v_{j, t} \quad$ Square of voltage at node $j$ 
$C_{s t, j, d}^{n o m}$ Final nominal capacity of battery systems for each node $j$ for each day $d$

$F_{i n j} \quad$ Function describing the cost of power injected into the feeder at the substation

$F_{\text {inv }} \quad$ Function describing the cost of investment of the battery nominal capacity and power

FO\&M Function describing the cost of operation and maintenance of the battery devices

$F_{p} \quad$ Function describing the cost of losses within the system including PV curtailment

$F_{s t, p} \quad$ Function describing the cost of battery losses due to charging/discharging efficiency

$N_{s t, j, d}^{n o m}$ The number of hours of nominal autonomy of the battery system at node $j$

$P_{s t, j, d}^{n o m}$ Final nominal power of battery systems for each node $j$ for each day $d$

$P_{0, t} \quad$ Active power flow at the substation

$P_{j k, t} \quad$ Active power of branch $j k$

$P_{p v, j, t}$ Photovoltaic injection at node $j$ at time $t$ after eventual curtailment

$P_{s t, j, t}$ Power injected by battery devices connected at node $j$

$Q_{j k, t} \quad$ Reactive power of branch $j k$

$Q_{p v, j, t} \mathrm{PV}$ reactive power injection at node $j$ after eventual curtailment

$S_{j k, t} \quad$ Apparent power of branch $j k$

$S_{p v, j, t} \mathrm{PV}$ apparent power flow at node $j$

$s o c_{s t, j, t}$ State of charge of the storage unit as a cumulation of energy at node $j$ and time step $t$

$V_{j, t} \quad$ Voltage at node $j$

\section{Introduction}

The increasing environmental concerns, is one of the main drivers behind the large scale development of distributed energy resources (DER) in electric distribution grids. This development involves connection of decentralized generators to the electric grid primarily photovoltaic (PV) and wind turbines and also micro-hydroelectric generators bring about new challenges for the distribution grid operators. Decentralized renewable energy generators can introduce bi-directional flow within the network, while their production is uncertain and variable due to its inherent dependence on weather conditions. Other specific challenges of the distribution grid include higher uncertainty due to reduced aggregation effects of DER generators, voltage profile deviation and increased power flow in electric lines. These challenges are generally localized therefore creating local voltage perturbations that may not be visible by the distribution operator.

Solutions to these challenges include infrastructure upgrades such as electric line reinforcement or automation and integration of smart grid functionalities such as on-line tap changers (OLTC), DER generation curtailment, storage devices, demand side management (DSM) [1]. Specific technologies related to flexibilities include privately owned grid connected batteries such as electric vehicles [2 or larger grid operator owned storage used to improve overall economic exploitation of the feeder. Demand side management optimization in smart grids and efficient smart grid technologies have been thouroughly explored for a variety of use cases [3, 4] [5] 6] 6]. Infrastructure upgrades are easily quantified. However, new control and flexibility functionality is difficult to quantify economically and integrate into the planning phase of distribution grids. The cost benefit analysis of varying smart grid technologies and management strategies will become more important as DER penetration increases in future distribution grid systems.

Grid storage elements are presented in the literature as a cost effective solution to deal with the above challenges in distribution grids with high DER penetration. A techno-economic analysis of energy storage elements as a solution to intermittency of DER is presented in [8]. That paper details the cost-effectiveness of different grid storage applications including regulation of transmission and distribution power quality, voltage regulation and control, energy management, smoothing of intermittent renewable energy production, energy back-up, peak shaving, etc. For each specific application, taking into account the operational strategy of the storage device is important when sizing and placing the unit.

The optimal sizing and placement of storage devices in distribution grids has been addressed through various mathematical modeling methods presented in the literature. The problem of calculating the optimal placement and size of storage devices of an electric grid is highly dimensional and non-convex. The resolution of this highly dimensional non-convex problem has been successful with multiple mathematical techniques including analytical techniques, classical techniques, artificial intelligence techniques and other miscellaneous techniques [9]. In a different review of energy storage allocation, four main categories are defined to solve this highly dimensional non-convex problem: analytical methods, mathematical programming, exhaustive search and heuristics [10]. The different existing methods are of different complexity, some being simple, i.e. based on an energy balance of the examined system to size the storage. However, for grid connected systems the placement involves analysis of the impact of storage 
devices to the grid. For this reason, techniques based on mathematical programming such as power flow and optimal power flow (OPF) are more appropriate. These methods can be used to simulate distribution system functionality with generators and storage devices while taking into account grid constraints as seen in [11]. OPF algorithms are capable of taking into account decision variables and therefore capable of analyzing active management of distribution grids. An example of an OPF that analyzes hosting capacity of an active distribution grid is found in [12], where curtailment strategies and dynamic line rating are explored to increase renewable energy penetration.

OPF algorithms are efficient at analyzing active distribution networks for operation and planning. The two primary problem resolution techniques for solving this highly dimensional non-convex problem include heuristic techniques or linear convex relaxations of the power flow equations. Heuristic algorithms have been used to solve the optimal placement and sizing of storage devices. For example, a two-step process with a master and a sub-problem is proposed in [13. This method firstly uses a heuristic algorithm to solve optimal placement and sizing of batteries. Secondly, a daily AC OPF multi-objective function takes into account optimal voltage control, minimization of network losses and total energy costs. Another paper presents a comprehensive sizing and siting algorithm using particle swarm optimization [14]. A different type of heuristic method was used to simultaneously size and place storage units using an artificial bee colony algorithm with an objective function that forces each storage node to be as autonomous as possible [15. Another heuristic method used to analyze grid connected storage for a multi-objective problem addressing both distribution grid and transmission grid objectives is found in [16]. However, heuristic algorithms often require a larger calculation burden and are not guaranteed to converge to a global optimal solution as noted in [17. A mixed integer linear programming approach for complete DER portfolio sizing and placement is presented in [18]. The mixed integer strategy uses linearized power flow equations and loss estimations. Mixed integer linear approximations are proven to be effective at solving the non-convex placement and sizing problem however the calculation time is high and scalability to large network sizes have not been addressed.

Convex relaxations of the power flow equations generally have a lower calculation burden. The relaxation of the power flow equation into a second order cone has already been theoretically explained and detailed mathematically in [19]. Papers addressing specifically optimal sizing and placement of storage devices using convex relaxations can be found in the literature. An impedance model was used to perform optimal placement and sizing in [20]. A convex relaxation was used for optimal placement and sizing of batteries with a linearized DC power flow for transmission planning with a maximum investment cost 21]. This linearization is not accurate for the high $\mathrm{R} / \mathrm{X}$ ratio found in the low voltage distribution sys- tems, which implies electrical losses that are non linear. The use of an AC OPF for optimal placement [22] or optimal sizing [23] are also found in the literature. In [24] the authors explore a two-step process of sizing and placement of storage units through relaxed power flow equations. However, this sizing methodology calculates power and energy imbalances locally at PV nodes and sizes the battery systems to mitigate these imbalances. Therefore, this methodology sizes the battery systems to reduce PV injection when power quality issues become an issue. This sizing methodology does not compare the cost of storage elements to other cost-effective solutions such as curtailment. The algorithm also does not analyze the possible benefits of batteries participating in an electricity market. A second order cone program (SOCP) OPF algorithm is then used in the second step to site the sized battery systems.

While there are multiple sizing and placement algorithms (e.g. [13, [14, 25, [16]), the objective function of each presented methodology varies significantly implying significant variation in the results of the optimization simulations. For example in 13 the objective is composed of minimizing the energy injection, voltage deviation and network losses. In 14 the objective is peak load shaving, improving voltage profile quality and providing active power adjustment capacity. In 25] the investment, replacement and operations costs of $\mathrm{PV}$, diesel generators, and battery banks as well as slack bus power, cost of energy not served, losses and excess energy of HPVDS. In [16] the cost of local generation, the cost of centralized generation, unit cost of storage and the unit cost of power conversion. Due to the fact that the exploitation of a battery system for a specific purpose can have significant effects on the optimal size and placement as stated in [8], the results of the sizing and placement of these studies are very difficult to compare.

The SOCP relaxation of the power flow equations is present in multiple articles in the literature for example [26]. However, it has been proven to be inexact during periods of high penetration from decentralized production. An example of this could be high PV production and low loads during the summer season. In order to overcome the challenge of inaccurate convex relaxations [27] presents an AC OPF algorithm that integrates linear cuts implemented in an iterative manner to ensure an exact and feasible relaxation of the power flow equations. In a followup work, this algorithm has then been developed into a multi-temporal one in order to more objectively evaluate the benefits of grid connected storage and other temporally dependent variables in [28].

This paper proposes a novel methodology to simultaneously perform optimal sizing and placement of storage devices to the distribution grid from a techno-economical view by considering the investment cost of batteries weighted against the operational benefit. Operational control of active power of storage and PV inverters are modeled with a multi-temporal OPF. The objective function of the op- 


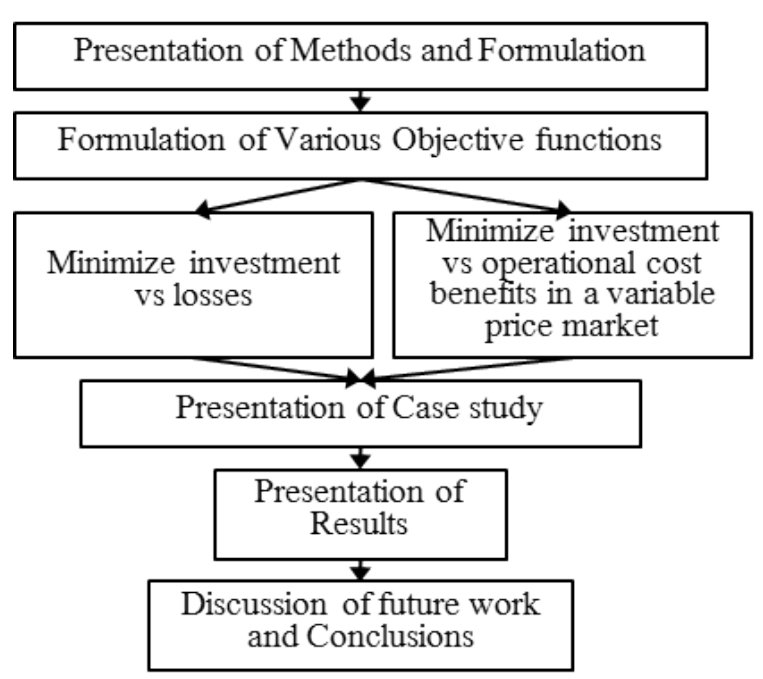

Figure 1: Paper organization

timization problem is formulated in a way to include economic operational benefit and constraints that guarantee power quality.

This methodology is capable of taking into account in detail the operational strategy of storage devices in order to make planning decisions about their sizing and placement. Therefore, it is effective for distribution grid planning applications with predefined operational strategies. The structure of this paper includes section II describing the optimal power flow algorithm, section III illustrating a case study of the proposed methodology and section IV stating significant conclusions. A flow chart of the papers organization is found in 1

\section{Optimal power flow model}

The proposed methodology relies on solving the optimization problem given by eqs. (1)-15. The objective function is the sum of the battery investment costs, operation and maintenance costs, the system losses and power imported at the feeder substation. The constraints of this model include the active power limits of PV systems defined by the maximum available power as a function of weather conditions eq. (2), apparent power limits of PV systems eqs. (3) and (4), power flow equations eqs. (5)(7), relaxation of the current equation eqs. (8) and (9) to represent the power flow equations as a convex SOCP, voltage limits of each branch eq. (10), battery state of charge (SOC) constraint eq. (11) and daily nominal power and capacity value constraints eqs. (12)-14). Constraint eq. (15) limits the ratio of nominal power and nominal capacity to be appropriate for distribution grid storage elements managed on a daily basis. This constraint also allows for fast convergence of the algorithm.

$$
\min F_{i n v}+F_{O \& M}+F_{p}+F_{s t, p}
$$

subject to:

$$
\begin{aligned}
& 0 \leq P_{p v, j, t} \leq \bar{P}_{p v, j, t} \\
& S_{p v, j, t} \leq \bar{S}_{p v, j, t} \\
& S_{p v, j, t}=\sqrt{P_{p v, j, t}^{2}+Q_{p v, j, t}^{2}} \\
& P_{i j, t}=P_{l d, j, t}+\sum_{j=0}^{J} P_{j k, t}+r_{i j} \ell_{i j, t}+P_{p v, j, t}+P_{s t, j, t} \\
& Q_{i j, t}=Q_{l d, j, t}+\sum_{j=0}^{J} Q_{j k, t}+x_{i j} \ell_{i j, t}+Q_{p v, j, t} \\
& v_{j, t}=v_{i, t}-2\left(r_{i j} P_{i j, t}+x_{i j} Q_{i j, t}\right)+\left(r_{i j}^{2}+x_{i j}^{2}\right) \ell_{i j, t} \\
& S_{i j, t} \geq \sqrt{P_{i j, t}^{2}+Q_{i j, t}^{2}} \\
& S_{i j, t} \geq \ell_{i j, t} v_{i, t} \\
& \underline{V}^{2} \leq v_{j, t} \leq \bar{V}^{2} \\
& s o c_{s t, j, t}=s o c_{s t, j, t-1}-t\left(P_{s t, j, t}+\eta_{s t}\left|P_{s t, j, t}\right|\right) \\
& P_{s t, j, d}^{n o m} \geq\left|P_{s t, j, t}\right| \\
& C_{s t, j, d}^{n o m} \geq s o c_{s t, j, t} \\
& C_{s t, j, d}^{n o m}=N_{s t, j, d}^{n o m} P_{s t, j, d}^{n o m} \\
& 0.1 \leq N_{s t, j, d}^{n o m} \leq 8
\end{aligned}
$$

The optimal sizing and placement of storage requires the resolution of a temporal and spatial problem. The temporal problem implies a coupling of multiple time steps to ensure coherence of the battery state of charge (SOC) between each consecutive time step. The spatial problem implies the consideration of all nodes as possible placement locations for storage devices. The multi-temporal OPF is already high dimensional. For a grid with 137 nodes, feasibility testing showed that up to 130 coupled time steps was returned results by the solver, any larger coupling returned a maximum size exceeded error. Therefore, a certain decoupling is necessary in order to complete an annual analysis. In this paper, a daily decoupling was implemented. Therefore, the number $\mathrm{T}$ of coupled timesteps is 24. A temporal decoupling is applied due to the size limitations of the optimization problem. The decoupling was chosen to be done on a daily basis due to the fact that battery systems are often managed on a daily basis. The coupled time steps of a one day period were then simulated for each day of the year in order to successfully complete an annual analysis. An additional constraint is added to avoid daily accumulation effects by forcing the state of charge (SOC) of the first and last time step of a day to be equal as stated in eq. (16).

$$
s o c_{s t, j, 0}=s o c_{s t, j, T}
$$

Constraint 14 is the product of two variables and is non-convex rendering the problem NP-hard. The variable 
$\mathrm{N}$ represents the number of hours of autonomy of the battery system, limited by feasible battery sizes of 0.1 to 8 . This relationship between the nominal power and capacity of a battery system is an essential relationship for accurately representing the cost of battery systems. In order to keep this constraint, a linearization of is performed through an iterative process. The linearization is shown in equation 17.

$$
N_{s t, j, d}^{n o m} P_{s t, j, d}^{n o m}=\frac{1}{2}\left[N_{0, j, d}^{n o m} * P_{s t, j, d}^{n o m}+N_{s t, j, d}^{n o m} * P_{0, j, d}^{n o m}\right]
$$

Where $N_{0, j, d}^{n o m}$ and $P_{0, j, d}^{n o m}$ are initial values assigned. With these initial values $P_{s t, j, d}^{n o m}$ and $N_{s t, j, d}^{n o m}$ are calculated with the algorithm. If the difference between the initial estimation and the final calculated values is larger than a 0.001 for either $P_{s t, j, d}^{n o m}$ or $N_{s t, j, d}^{n o m}$ a new iteration is performed assigning $N_{0, j, d}^{n o m}$ and $P_{0, j, d}^{n o m}$ to the values of $P_{s t, j, d}^{n o m}$ or $N_{s t, j, d}^{n o m}$.

The algorithm effectively calculates the optimal size and placement of storage devices for each node for each day. This sizing and placement exercise therefore results in 365 optimal nominal capacity and power. The final optimal size must then be choosen from analyzing these 365 values. This is done by taking the 75 th quantile of the set of optimal values to calculate a final annual optimal size.

\subsection{Variations of the objective function}

The objective function of the general form given by Eq. eq. (1) can be altered in order to size the battery systems for different purposes. Two objectives are considered in this Section. The first objective function is to size the battery systems to minimize losses in the system section. The second possible objective function considers the minimization of losses and the absolute value of the active power injection from the high voltage grid to the medium voltage grid at the substation.

\subsubsection{Loss minimization}

The first sizing exercise entails using the battery system only for loss minimization and allows a comparison between loss minimization eq. (21), losses associated with charging/discharging of the battery systems eq. 22 and the sum of battery investment costs eq. 19 and battery operations costs eq. 20. The objective function is therefore eq. 18). The optimal nominal power and capacity is then calculated based only on the economic viability of using batteries for loss reduction.

$$
\min F_{i n v}+F_{O \& M}+F_{p}+F_{s t, p}
$$

where:

$$
F_{i n v}=\sum_{j=0}^{J} c_{s t}^{i n v} C_{s t, j, d}^{n o m}
$$

$$
\begin{aligned}
& F_{O \& M}=\sum_{j=0}^{J} c_{s t}^{o m} t P_{s t, j, d}^{n o m} \\
& F_{p}=\sum_{t=0}^{T} \sum_{j=0}^{J} c_{e, t} r_{i j} \ell_{i j, t}+c_{e, t} t\left[\bar{P}_{p v, j, t}-P_{p v, j, t}\right] \\
& F_{s t, p}=\sum_{t=0}^{T} \sum_{j=0}^{J} c_{e, t} \eta_{s t} t\left|P_{s t, j, t}\right|
\end{aligned}
$$

subject to:eqs. 2 15

The losses considered are the line losses, PV curtailment and the losses of the battery system due to the battery charging efficiency. If the sum of the operational costs and the investment costs of the battery systems is higher than the economic gain from loss reduction, the algorithm eq. (18) will calculate zero nominal capacity and power for each node.

\subsubsection{Minimization of absolute active power flow at sub- station}

Battery systems can also be used to participate in variable pricing electricity markets. This implies using battery systems to buy and sell electricity from the grid based on the hourly price of electricity. This objective function as detailed in eq. 23 allows the calculation of economic gains through battery participation in variable pricing markets and encourages autonomy of the feeder by minimization of the total absolute active power flow imported at the substation $\left|P_{0, t}\right|$. This formulation does not include explicitly the losses associated with the battery charging efficiency because this energy is already counted in the variable $\left|P_{0, t}\right|$.

$$
\min _{j \in J} F_{i n v}+F_{O \& M}+F_{p}+F_{i n j}
$$

where:

$$
F_{i n j}=\sum_{t=0}^{T} c_{e, t} t\left|P_{0, t}\right|
$$

subject to:eqs. 2 2 - 15

These two objective function variations can be used to determine the size and placement of battery storage devices coupled with installed PV systems for specific enduse scenarios. This algorithm does not consider constraints to exclude very small battery systems. Therefore it is incapable of minimizing the number of nodes that battery systems are installed at. As a result the algorithm often sizes battery systems for every node. However, in some 


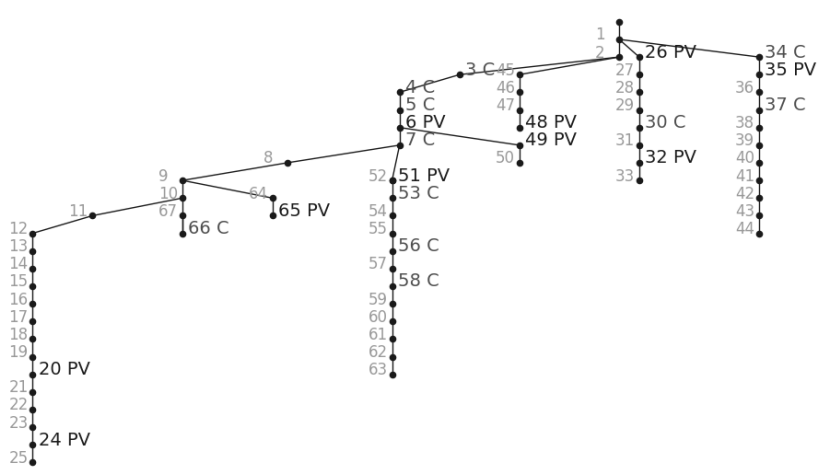

Figure 2: Grid topology including low voltage substation (C) and PV system placement (PV)

cases, it can be desirable by the DSO to consider a limited number of battery systems or a battery system size minimum. If a minimum battery size was introduced into the problem, it would introduce a binary constraint making the problem NP Hard. Therefore, to deal with these small infeasible systems sizes, an iterative approach can be taken. At a first stage, nodes with the larger battery sizes are identified with the initial sizing and placement algorithm. Then, final sizing can be performed with added constraints eqs. (25) and (26) as a second iteration.

$$
\begin{array}{lll}
C_{s t, j, d}^{n o m}=0 & \text { for } & J-J_{s t} \\
P_{s t, j, d}^{n o m}=0 & \text { for } & J-J_{s t}
\end{array}
$$

The criteria for choosing final node placement can be determined by choosing a maximum number of battery systems or an acceptable maximum and minimum size of battery systems. For sizing based on a maximum number of systems, $J_{s t}$ in eq. 26) represents the number of nodes $n$ with the $n$ largest values for the nominal capacity and power. For sizing based on an acceptable maximum and minimum size, $J_{s t}$ represents the nodes with feasible nominal power and capacity values.

\section{Results}

\subsection{Case study}

The example grid used for this study is a medium voltage distribution grid published here [29]. This grid is composed of 69 nodes with a nominal voltage of $12.66 \mathrm{kV}$ and is assumed to be located in Nice, France. A map of the grid topology can be found in Fig 2 .

\subsection{Generation and load profiles}

Electric load profiles were simulated using a load simulator as described in 30] for each low voltage substation load profile. Residential and commercial load profiles are simulated with statistically accurate representations of surface area, electric heating and number of individuals

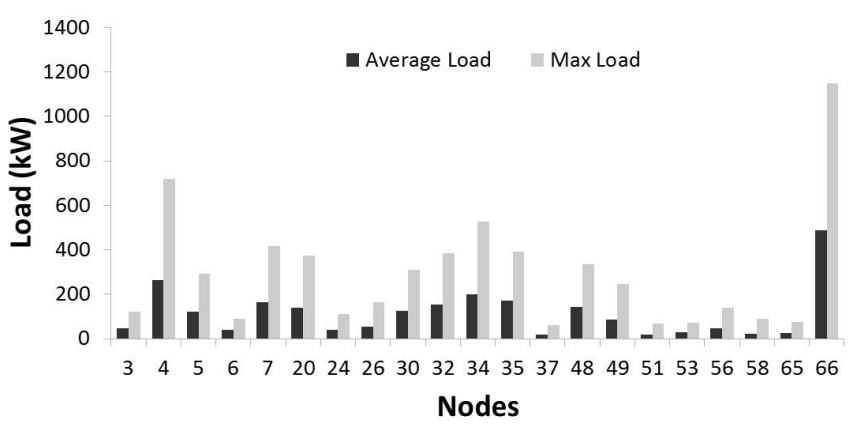

Figure 3: Load characteristics for all loaded nodes

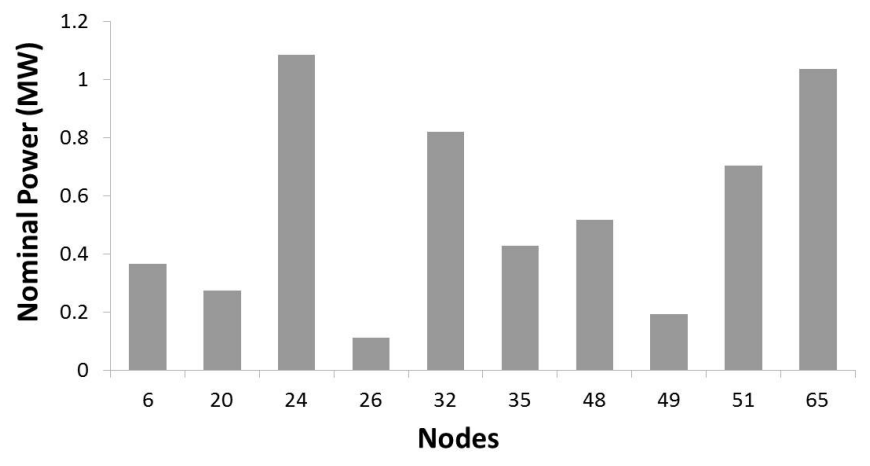

Figure 4: PV nominal power ratings for each $\mathrm{PV}$ node

that align with the INSEE household inventory database of France. The location of each load node was chosen randomly due to the fact that no grid load data was available. The medium voltage feeder is assumed to be a 10 MVA transformer serving 21 low voltage substations. Load profiles aligning with meteorological data in Nice, France indicated a peak load of 4.7 MW during the summer and 5.9 MW during the winter with an average load of respectively 2.1 MW and 2.6 MW. Solar radiation data was simulated for Nice, France for the year 2012 by analyzing the global irradiation collected by HelioClim 3 [31]. The PV system production was calculated based on a statistical distribution of direct and diffuse irradiation 32. This data is then integrated into a projection model to calculate the percentage of direct and diffuse irradiation exposed to the panels 33. A system performance coefficient is then calculated based on the atmospheric conditions extrapolated from a performance data base of PV systems in the south of France.

An amount of $10 \mathrm{PV}$ systems were randomly assigned to 10 nodes. The size of these systems was also chosen randomly to be between $125-1250 \mathrm{~kW}$. Characteristics of the electric load profile nodes can be found in Fig. 3 and PV size information can be found in Figure 4.

\subsection{Economic analysis}

In order to analyze the economic viability of battery systems, market price variation and battery system costs 


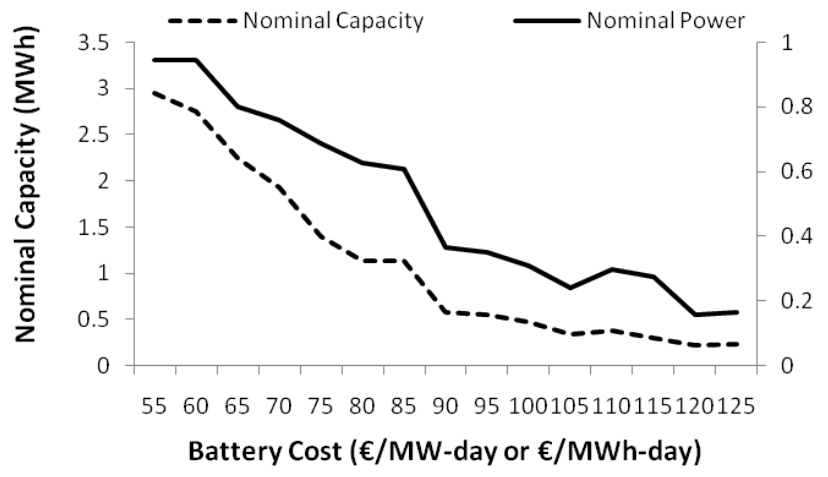

Figure 5: Total aggregated nominal capacity and power optimal system size as a function of battery costs

were taken into account. Historical market data from France was used for 2012.

The capital cost of batteries for nominal capacity and power is determined by analyzing the study 34. The nine case studies on high performance lead acid battery for transmission and distribution applications were analyzed to calculate the battery investment cost per MWh. Battery costs ranged from $500 \mathrm{k} € / \mathrm{MWh}$ to $2.5 \mathrm{M} € / \mathrm{MWh}$. To integrate these costs into the daily analysis, the investment costs per MWh are divided by the lifetime of the system. For all economic analysis, the battery life is assumed to be 10 years as assumed also in 35. Therefore, the "daily" investment costs ranged from 137.6 $€$ /MWh-day to 678.6 $€ / M W h-d a y$. Due to the large range of battery investment costs, a sensitivity analysis was completed to determine the price of batteries that is economically viable. In [35], operations and maintenance prices of the battery systems are given to be between $2-6$ cents per $\mathrm{kWh}$. For this study this cost is selected to be 2 cents/kWh.

\subsection{Results}

The two objective functions presented in eq. 18 and eq. (23) were analyzed to determine economically viable battery placement and sizing. In all considered scenarios, equation eq. (18) showed that battery investments were not economically viable for only loss minimization with the considered PV penetration and load profiles. A sensitivity analysis of the battery costs was performed with objective function eq. 23) to analyze the economic viability of storage systems used for loss minimization in addition to market participation. Results of the nominal power and capacity specifications calculated for each node using objective function eq. (23) can be found in Fig. 5 .

This sensitivity analysis compares the sum of total nominal power and capacity for a feeder in relation to different investment costs. These total nominal capacity and power values are a sum of the individual nominal capacity and power values for each node. The price of batteries calculated by this study to be economically viable are lower than the battery costs found in 34. For example, a battery with a capacity of $2 \mathrm{MWh}$ and a nominal power rating

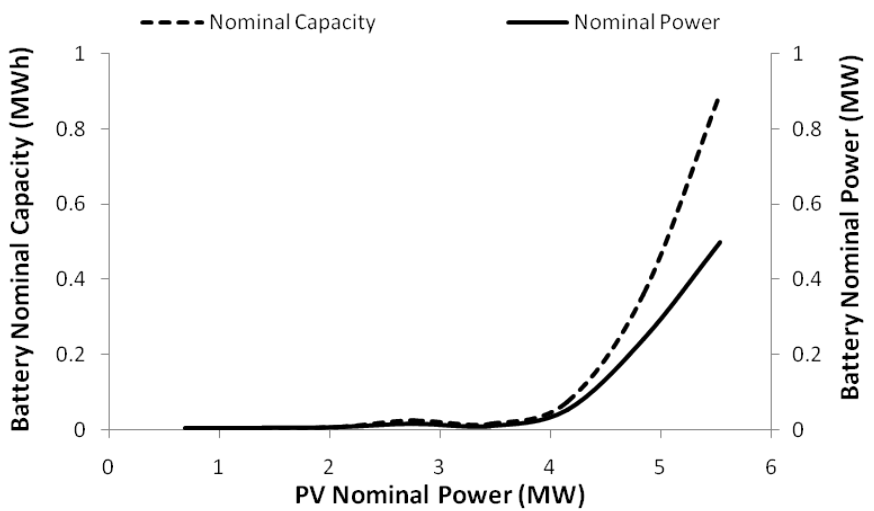

Figure 6: Sensitivity analysis of PV penetration in relation to optimal aggregated nominal power and capacity battery size for an investment cost of $85 € /$ MWh-day

of $1 \mathrm{MW}$ cost on average $2.238 \mathrm{M} €$ according to the study [34. In the sensitivity analysis, if the investment costs of the battery are $85 € /$ MWh-day, the total ideal nominal capacity and power for the feeder is $2 \mathrm{MWh}$ and $1 \mathrm{MW}$. This per day investment cost can be translated into an initial investment cost by taking into account a life time of 10 years. The calculated initial investment cost of this system is therefore $310.25 \mathrm{k} €$. This implies that for this battery to be economically viable, capital costs must be 7.2 times cheaper than the battery costs published in 34 .

For the specific example of a battery investment price of $85 €$ /MWh-day, a sensitivity analysis was performed comparing the PV penetration and associated battery size. This comparison is found in Fig. 6

This sensitivity analysis shows a high correlation between optimally sized battery systems and PV system size. Therefore grid connected battery systems become exponentially more economical with high penetration of DER. A comparison of centralized and decentralized optimally placed capacity is shown in Fig. 7. The centralized storage nodes are considered to be any node on the main branch of the grid topology tree. The list of centralized nodes for this network are therefore $0,1,2,3,4,5$ and 6 . All other nodes are considered to be decentralized placement nodes for storage devices.

As seen from this analysis, decentralized battery systems are prioritized for lower battery investment costs while centralized systems size is mostly stable over all investment costs. Decentralized battery systems are more prioritized when battery costs are low and overall total capacity and power installed is higher. When total capacity and power installed is smaller, the ratio of decentralized to centralized systems is also much smaller.

The partitioning of total nominal battery capacity and power as a function of battery investment cost is found in Fig. 8 to demonstrate the repartitioning of battery capacity and power.

As seen in 8, certain decentralized nodes including for example node 25, 34, 65 and 66 are prioritized for storage 


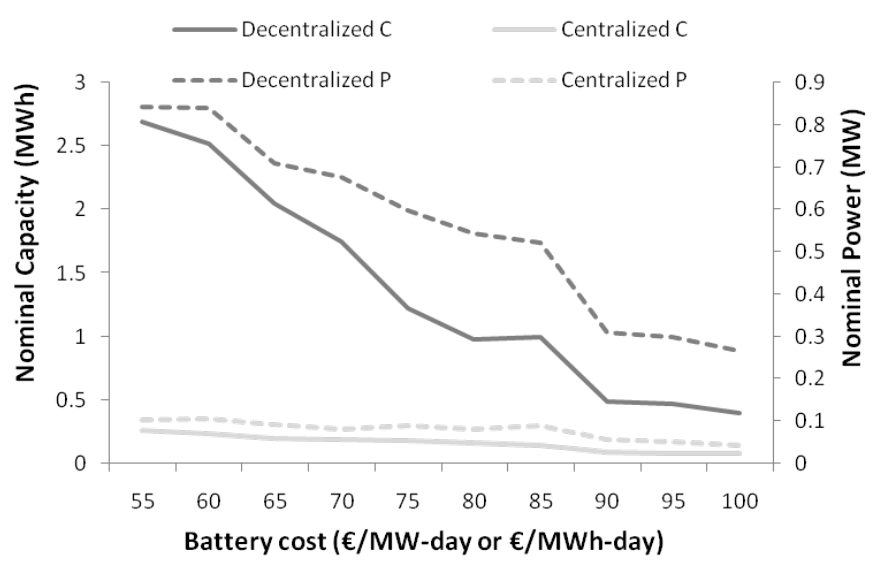

Figure 7: Comparison of centralized and decentralized nominal power $(\mathrm{P})$ and capacity $(\mathrm{C})$ optimal system size as a function of battery costs
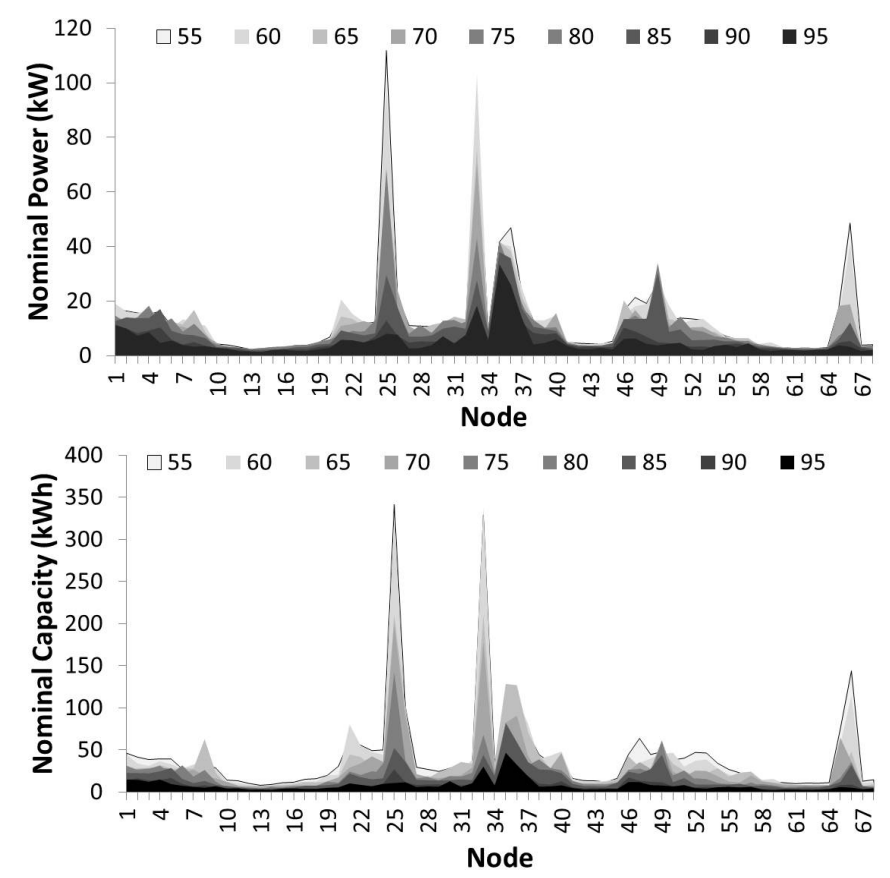

Figure 8: Calculated size of battery systems for each node including nominal power (upper) and nominal capacity (lower) with battery prices varying from $55 € /$ MWh-day to $95 € /$ MWh-day

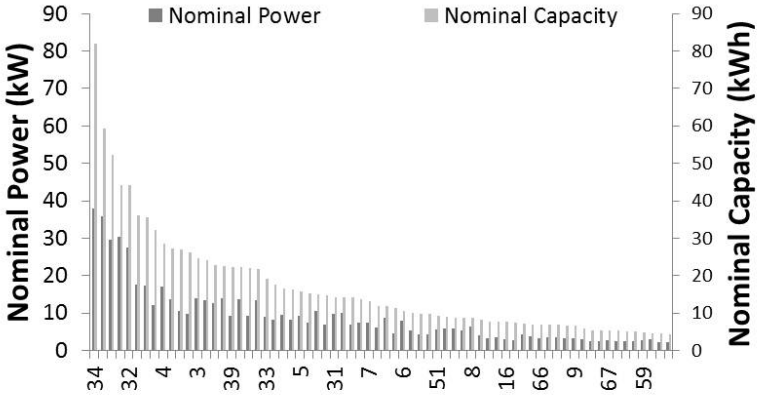

Node

Figure 9: Calculated size of battery sizes for battery prices of 85 $€ /$ MWh-day

placement. These three nodes give three different examples of when storage is advantages. Node 25 is a priority due to the high nominal PV power installed at node 24 and also the fact that this system is at the end of an electric feeder. For the case of node 34, high nominal PV power at node 35 combined with high loading at node 34 makes this node a priority. Node 65 and 66 are two nodes relatively close to each other. Node 66 has a high load and node 65 has a large PV system. The algorithm assigned capacity to both nodes, however in all cases a larger capacity is assigned to node 65 than 66 prioritizing a placement closer to the PV system installation rather than the highly loaded node.

A closer look is taken into the case study with a battery cost of $85 € / M W h-d a y$. The individual calculated sizes of battery systems for each node can be found in Fig. 9

In Fig. 9 a nominal power and capacity value of storage devices is assigned to every node. This is due to the fact that many small systems allow to reduce losses and regulate the balanced of generation and consumption at each node. The size of the battery systems exponentially decreases when the nodes are ranked by size. This shows that certain nodes are high priority but having a storage at all nodes is ideal.

The objective function does not penalize small battery systems, therefore the infeasible small battery systems must be eliminated through an iterative qualitative analysis. The final optimal placement and sizing of battery systems can be calculated by limiting the possible nodes where battery systems can be placed. This selection is performed based on the initial sizing and placement analysis. For the case defined by $85 € / M W h$-day, the total number of systems installed was fixed to be 10 . Therefore, a final analysis is performed by using eq. (26) and setting fixed the set $J_{s t}$ as seen in eq. (27). The final resulting ideal sizing is found in Fig. 10.

$$
J_{s t}=4,34,48,35,36,32,47,25,24,65
$$

After fixing the maximum number of battery systems to be 10 , a similar total nominal power and capacity is 


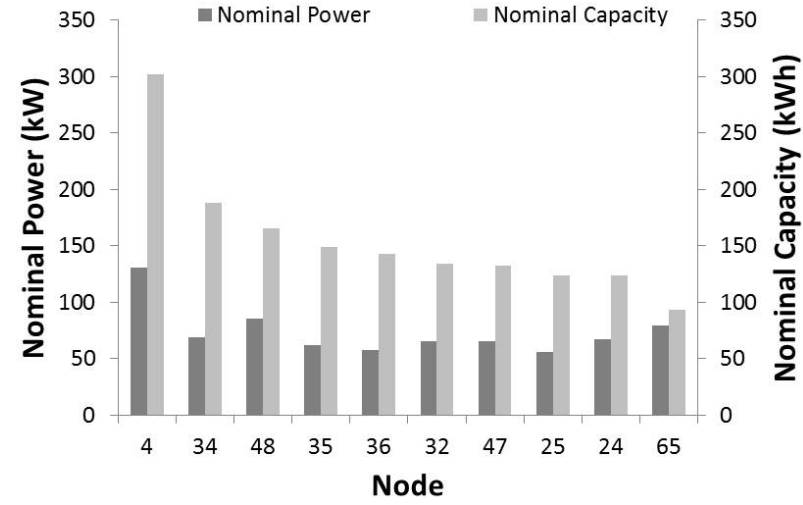

Figure 10: Final size of selected nodes with a battery cost of 85 $€ /$ MWh-day

Table 1: Operation of electrical feeder with and without battery integration. Annual analysis for the case of $85 € /$ MWh-day

\begin{tabular}{l|c|c}
\hline Characteristic & With battery & Without battery \\
\hline $\begin{array}{l}\text { Total Cost of En- } \\
\text { ergy Imports (thou- } \\
\text { sand } € \text { ) }\end{array}$ & 467 & 489 \\
\hline $\begin{array}{l}\text { Total Price of En- } \\
\text { ergy Exports (thou- } \\
\text { sand } € \text { ) }\end{array}$ & 1.64 & 2.13 \\
\hline $\begin{array}{l}\text { PV Benefits (thou- } \\
\text { sand } € \text { ) }\end{array}$ & 334 & 314 \\
\hline $\begin{array}{l}\text { PV Curtailement } \\
\text { (MWh) }\end{array}$ & 1.05 & 1.39 \\
\hline $\begin{array}{l}\text { Nominal PV Power } \\
\text { Installed (MW) }\end{array}$ & 5.53 & 5.53 \\
\hline $\begin{array}{l}\text { Nominal Batt Power } \\
\text { Installed (MW) }\end{array}$ & 0.74 & 0.0 \\
\hline $\begin{array}{l}\text { Nominal Batt Ca- } \\
\text { pacity Installed } \\
\text { MWh) }\end{array}$ & 1.55 & 0.0 \\
\hline
\end{tabular}

sized by the algorithm. Due to limited number of nodes for the repartition, the size of each system is therefore significantly larger than when all nodes are considered. Annual analysis results are then calculated by fixing the upper bounds of the nominal power and capacity of each battery system based on the values graphed in Fig. 10 and calculated by fig. 10 . The feeder operation with and without battery systems is shown in Table 1 .

The operation of the above feeder is affected by the presence of optimally placed and sized battery systems that allow for load shifting through pricing signals. The sum of imports and exports at the substation is therefore decreased. The first line in Table 1 shows that the optimized battery systems succeed to decrease the cost of imported energy. The level of exported energy shown in line two remains low due to the objective function that
Table 2: Calculation time for daily and annual analysis

\begin{tabular}{c|c|c}
\hline Algorithm & $\begin{array}{c}\text { Simulated } \\
\text { time steps }\end{array}$ & $\begin{array}{c}\text { Calculation } \\
\text { time }(\mathrm{s})\end{array}$ \\
\hline $\begin{array}{c}\text { multi-temporal } \\
\text { SOCP OPF }\end{array}$ & 24 & 2.7 \\
\hline $\begin{array}{c}\text { multi-temporal } \\
\text { SOCP OPF }\end{array}$ & $24 \times 365$ & 2700 \\
\hline
\end{tabular}

minimizes the absolute energy flow at the feeder. Line four shows a reduction in PV curtailment due to added battery systems therefore increasing PV benefits as shown in line three.

\subsection{Algorithmic performance}

The algorithmic performance of the simulation of a single time step and the full annual analysis has been observed as shown in Table 2 .

The iterative approach to calculate $P_{s t, j, d}^{n o m}$ and $N_{s t, j, d}^{n o m}$ increases the daily analysis calculation time linearly depending on the number of iterations needed for convergence. The calculation time showed in 2 are therefore averages of all analysis performed.

\section{Discussion}

This algorithm is capable of calculating the placement and sizing of storage devices in a distribution grid. The size is sensitive to the investment cost of the batteries as shown by the sensitivity analysis. In the context of a project, the capital investment of the project may be lower than the ideal size of storage capacity and power. If an investment constraint exists, an investment constraint can be integrated into the optimization problem as seen in eq. 28.

$$
c_{s t}^{i n v} \sum_{j=0}^{J} C_{s t, j, d}^{n o m} \leq I^{\max }
$$

This algorithm does not consider the planning of active demand as an alternative solution to electrochemical storage. While the algorithmic structure is very similar to modeling electrochemical storage elements, the calculation of the cost of demand side management is difficult. The costs are non-linear, client-dependent and data sets quantifying these costs are rare. Other possible solutions that were not explored include infrastructure upgrades.

A challenge identified in this paper was the capability to integrate non linear cost functions with respect to the nominal power and capacity of batteries. Another intersting improvement to the cost function could include the integration of variable battery technologies and the associated variable cost parameters. Future work can be done to integrate these non-linear characteristics into the SOCP convex relaxation algorithm. 


\section{Conclusion}

This paper has successfully demonstrated an adaptation of a SOCP convex relaxation of the power flow equations for optimal sizing and placement of battery systems in a medium voltage distribution feeder. The proposed algorithm that simultaneously sizes and places battery systems can be effectively used to analyze the economic viability of operational case studies in comparison to investment and operational costs. The specific contributions of this paper include:

- A high performance algorithm for solving simultaneously the sizing and placement problem through decomposition into daily analysis and assessment of a typical year of operation of the system under study and taking into account the impact of the electrical network. This is a major contribution compared to the state of the art where these two problems of placement and sizing are often considered in a decoupled way

- A methodology to integrate operational case studies of battery management strategies into the planning phase of active distribution grids

- A qualitative study of battery investment costs and their operational benfits to make investment decisions about grid connected storage

- A demonstration of the increasing benefit of grid connected storage in the presence of high DER penetration

This type of innovative algorithm gives insights into the advantages of grid connected storage devices in distribution systems and the integration of operational strategies into the planning phase.

\section{Acknowledgment}

The authors would like to thank Mr. Thibaut Barbier for his support with the simulated load curves. Authors would like to thank Dr. Miguel Heleno from Lawrence Berkeley National Lab for useful discussions on possible comparative studies and existing sizing and placement tools. This work is performed in the frame of the Horizon 2020 project Sensible (Grant Agreement 645963) funded in part by the European Commission under the Horizon 2020 Framework Program as well as a PhD scholarship grant supported by the French Energy agency ADEME (Agence de l'Environnement et de la Matrise de l'Energie).

\section{References}

[1] M. L. Tuballa, M. L. Abundo, A review of the development of smart grid technologies, Renewable and Sustainable Energy Reviews 59 (2016) 710 - 725. doi:http://dx.doi.org/10.1016/ j.rser.2016.01.011.

URL http://www.sciencedirect.com/science/article/pii/ S1364032116000393
[2] M. H. Amini, M. P. Moghaddam, O. Karabasoglu, Simultaneous allocation of electric vehicles parking lots and distributed renewable resources in smart power distribution networks Sustainable Cities and Society 28 (2017) $332-342$. doi:https: //doi.org/10.1016/j.scs.2016.10.006

URL http://www.sciencedirect.com/science/article/pii/ S2210670716304966

[3] A. Ogunjuyigbe, T. Ayodele, O. Akinola, User satisfactioninduced demand side load management in residential buildings with user budget constraint, Applied Energy 187 (2017) 352 - 366. doi:http://dx.doi.org/10.1016/j.apenergy.2016.11. 071

URL http://www.sciencedirect.com/science/article/pii/ S0306261916317020

[4] F. Kamyab, M. Amini, S. Sheykhha, M. Hasanpour, M. M. Jalali, Demand response program in smart grid using supply function bidding mechanism, IEEE Transactions on Smart Grid 7 (3) (2016) 1277-1284. doi:10.1109/TSG. 2015.2430364.

[5] H. Esen, M. Inalli, M. Esen, Technoeconomic appraisal of a ground source heat pump system for a heating season in eastern turkey Energy Conversion and Management 47 (9) (2006) 1281 - 1297. doi:http://dx.doi.org/10.1016/j.enconman. 2005.06.024

URL http://www.sciencedirect.com/science/article/pii/ S0196890405001755

[6] H. Esen, M. Inalli, M. Esen, A techno-economic comparison of ground-coupled and air-coupled heat pump system for space cooling, Building and Environment 42 (5) (2007) 1955 - 1965. doi:http://dx.doi.org/10.1016/j.buildenv.2006.04.007 URL http://www.sciencedirect.com/science/article/pii/ S0360132306000977

[7] M. Esen, T. Yuksel, Experimental evaluation of using various renewable energy sources for heating a greenhouse, Energy and Buildings 65 (2013) 340 - 351. doi:http://dx.doi.org/10. 1016/j.enbuild.2013.06.018

URL http://www.sciencedirect.com/science/article/pii/ S0378778813003563

[8] X. Luo, J. Wang, M. Dooner, J. Clarke, Overview of current development in electrical energy storage technologies and the application potential in power system operation, Applied Energy 137 (2015) 511 - 536. doi:http://dx.doi.org/10.1016/ j.apenergy.2014.09.081

[9] P. Prakash, D. K. Khatod, Optimal sizing and siting techniques for distributed generation in distribution systems: A review Renewable and Sustainable Energy Reviews 57 (2016) 111 130. doi:http://dx.doi.org/10.1016/j.rser.2015.12.099 URL http://www.sciencedirect.com/science/article/pii/ S1364032115014823

[10] M. Zidar, P. S. Georgilakis, N. D. Hatziargyriou, T. Capuder, D. krlec, Review of energy storage allocation in power distribution networks: applications, methods and future research, IET Generation, Transmission Distribution 10 (3) (2016) 645-652. doi:10.1049/iet-gtd.2015.0447

[11] K. G. Boroojeni, M. H. Amini, A. Nejadpak, S. S. Iyengar, B. Hoseinzadeh, C. L. Bak, A theoretical bilevel control scheme for power networks with large-scale penetration of distributed renewable resources, in: 2016 IEEE International Conference on Electro Information Technology (EIT), 2016, pp. 0510-0515. doi:10.1109/EIT.2016.7535293.

[12] N. Etherden, M. Bollen, Increasing the hosting capacity of distribution networks by curtailment of renewable energy resources, in: Proceedings of 2011 IEEE PowerTech, Trondheim, Norway, 2011, pp. 1-7. doi:10.1109/PTC.2011.6019292

[13] M. Nick, M. Hohmann, R. Cherkaoui, M. Paolone, Optimal location and sizing of distributed storage systems in active distribution networks, in: Proceedings of 2013 IEEE PowerTech Conference, Grenoble, France, 2013, pp. 1-6. doi:10.1109/ PTC.2013.6652514

[14] Z. Qing, Y. Nanhua, Z. Xiaoping, Y. You, D. Liu, Optimal siting amp; sizing of battery energy storage system in active distribution network, in: IEEE PES ISGT Europe 2013, 2013, 
pp. 1-5. doi:10.1109/ISGTEurope.2013.6695235

[15] A. El-Zonkoly, Optimal placement and schedule of multiple grid connected hybrid energy systems, International Journal of Electrical Power \& Energy Systems 61 (2014) $239-247$. doi:http://dx.doi.org/10.1016/j.ijepes.2014.03.040

[16] M. Motalleb, E. Reihani, R. Ghorbani, Optimal placement and sizing of the storage supporting transmission and distribution networks $\quad$ Renewable Energy 94 (2016) 651 - 659. doi:http: //dx.doi.org/10.1016/j.renene.2016.03.101

URL http://www.sciencedirect.com/science/article/pii/ S0960148116302853

[17] S. Paudyal, C. Canizares, K. Bhattacharya, Three-phase distribution opf in smart grids: Optimality versus computational burden, in: Innovative Smart Grid Technologies (ISGT Europe), 2011 2nd IEEE PES International Conference and Exhibition on, 2011, pp. 1-7. doi:10.1109/ISGTEurope.2011.6162628

[18] S. Mashayekh, M. Stadler, G. Cardoso, M. Heleno, A mixed integer linear programming approach for optimal der portfolio, sizing, and placement in multi-energy microgrids Applied Energy 187 (2017) 154 - 168. doi:http://dx.doi.org/10.1016/ j.apenergy.2016.11.020

URL http://www.sciencedirect.com/science/article/pii/ S0306261916316051

[19] L. Gan, S. H. Low, Convex relaxations and linear approximation for optimal power flow in multiphase radial networks, in: Power Systems Computation Conference (PSCC), 2014, 2014, pp. 1-9. doi:10.1109/PSCC.2014.7038399

[20] H. Nazaripouya, Y. Wang, P. Chu, H. R. Pota, R. Gadh, Optimal sizing and placement of battery energy storage in distribution system based on solar size for voltage regulation, in: 2015 IEEE Power Energy Society General Meeting, 2015, pp. 1-5. doi:10.1109/PESGM.2015.7286059

21] C. Thrampoulidis, S. Bose, B. Hassibi, Optimal placement of distributed energy storage in power networks, IEEE Transactions on Automatic Control 61 (2) (2016) 416-429. doi: 10.1109/TAC. 2015.2437527

[22] M. Ghofrani, A. Arabali, M. Etezadi-Amoli, M. S. Fadali, A framework for optimal placement of energy storage units within a power system with high wind penetration, IEEE Transactions on Sustainable Energy 4 (2) (2013) 434-442. doi: 10.1109/TSTE.2012.2227343

[23] I. Sharma, K. Bhattacharya, Optimal sizing of battery energy storage systems in unbalanced distribution feeders, in: Industrial Electronics Society, IECON 2013 - 39th Annual Conference of the IEEE, 2013, pp. 2133-2138. doi:10.1109/IECON.2013. 6699461

[24] Q. Li, R. Ayyanar, V. Vittal, Convex optimization for des planning and operation in radial distribution systems with high penetration of photovoltaic resources, IEEE Transactions on Sustainable Energy 7 (3) (2016) 985-995. doi:10.1109/TSTE. 2015. 2509648

[25] A. El-Zonkoly, Optimal placement and schedule of multiple grid connected hybrid energy systems, International Journal of Electrical Power \& Energy Systems 61 (2014) 239 - 247. doi:http://dx.doi.org/10.1016/j.ijepes.2014.03.040

[26] J. F. Marley, D. K. Molzahn, I. A. Hiskens, Solving multiperiod opf problems using an ac-qp algorithm initialized with an socp relaxation, IEEE Transactions on Power Systems 32 (5) (2017) 3538-3548. doi:10.1109/TPWRS.2016.2636132.

[27] S. Abdelouadoud, R. Girard, F. Neirac, T. Guiot, Optimal power flow of a distribution system based on increasingly tight cutting planes added to a second order cone relaxation, International Journal of Electrical Power \& Energy Systems 69 (2015) 9 - 17. doi:http://dx.doi.org/10.1016/j.ijepes.2014.12. 084

[28] E. Grover-Silva, R. Girard, G. Kariniotakis, Multi-temporal optimal power flow for assessing the renewable generation hosting capacity of an active distribution system, in: Proceedings of 2016 IEEE PES Transmission and Distribution Conference and Exposition (T D), Dallas, USA, 2016, pp. 1-5. doi:10.1109/TDC.2016.7520043.
[29] M. E. Baran, F. F. Wu, Optimal capacitor placement on radial distribution systems, IEEE Transactions on Power Delivery 4 (1) (1989) 725-734. doi:10.1109/61.19265

[30] T. Barbier, R. Girard, F. P. Neirac, N. Kong, G. Kariniotakis, A novel approach for electric load curve holistic modelling and simulation, in: Proceedings of 2014 IEEE MedPower Conference, Athens, Greece, 2014, pp. 1-8. doi:10.1049/cp.2014. 1704

[31] P. Blanc, B. Gschwind, M. Lefevre, L. Wald, The HelioClim Project: Surface Solar Irradiance Data for Climate Applications, Remote Sensing 3 (2) (2011) 343-361, uRL : http://www.mdpi.com/2072-4292/3/2/343/. doi:10.3390/ rs3020343

[32] J. Ruiz-Arias, H. Alsamamra, J. Tovar-Pescador, D. PozoVzquez, Proposal of a regressive model for the hourly diffuse solar radiation under all sky conditions, Energy Conversion and Management 51 (5) (2010) 881 - 893. doi:http: //dx.doi.org/10.1016/j.enconman.2009.11.024

[33] T. Muneer, Solar radiation and daylight models for the energy efficient design of buildings, Architectural Press, 1997.

[34] A. B. C. B. C. K. D. M. R. S. B. C. A. L. C. D. T. B. Abbas A. Akhil, Georgianne Huff, W. D. Gauntlett, Doe/epri electricity storage handbook in collaboration with nreca, Sandia Report (February 2015).

[35] G. C. Jim Eyer, Energy storage for the electricity grid: Benefits and market potential assessment guide, Sandia Report (February 2010). 